\title{
A SIMPLIFIED APPROACH FOR THE HYDRO-FORMING PROCESS OF BI-METALLIC COMPOSITE PIPE
}

In this article, it aims to propose effective approaches for hydro-forming process of bi-metallic composite pipe by assuming plane strain and elastic-perfectly plastic material model. It derives expressions for predicting hydro-forming pressure and residual stress of the forming process of bi-metallic composite pipe. Test data from available experiments is employed to check the model and formulas. It shows the reliability of the proposed model and formulas impersonally.

Keywords: metallic pipe, composite, hydro-forming, pressure, residual stress

\section{Introduction}

Currently, pipeline is widely used in petroleum, chemical engineering, metallurgy, electric power and city gas, heating and water supply systems, thus the safety of the pipeline is a problem due to the attendant corrosions, such problem is growing tremendously with the increasing use of pipeline.

A new type of pipeline, called bi-metallic composite pipe, has proposed to solve the corrosion problem these years, it composes of two different metal pipes, i.e., one kind of inner pipe, another outer pipe (base pipe). Both pipes are connected closely through deformation or other technique. The general idea for the design of such bimetallic composite pipe is that the base pipe is to withstand for the requirement of stress and the inner lining pipe material is to meet the demands of corrosion or abrasion. As a result, the composite pipe has the full advantages of both the base pipe and the liner pipe material, it has not only the high strength, but also excellent anti-corrosion, wear resistance and other properties, and it reduces production costs tremendously, saving the use of rare and noble elements. Thus the bi-metallic composite pipe could be widely used in petroleum, chemical engineering, nuclear industry, medical, food, construction, fire and other fields.

According to the manufacturing methods, the production of bi-metal composite pipe can be divided into plastic forming mode and non-plastic forming mode. In the plastic pipe forming technology, the pipe is plastic deformed locally or totally to perform the forming process of the inner tube and the outer tube; Currently, the plastic forming is widely used domestic and abroad since the plastic pipe forming technology has advantage of high efficiency with simple process and equipment. The plastic forming mode includes mainly mechanical drawing method, rolling method, hydro-forming method and explosive forming method.
The mechanical drawing and hydro-forming methods are the important process of bi-metallic composite pipe production. Therefore, it is important to analyze the deformation and stress conditions in the forming process of bi-metallic composite pipe by hydro-forming and drawing methods, so that rational and effective design of the production process could be conducted.

It involves rather complex plastic deforming process in hydraulic bulging and drawing processes for bi-metallic composite pipe manufacturing. Both physical and geometric nonlinearity is contained, in addition to the complex boundary conditions [1-3], which make it difficult to establish a rigorous theoretical analysis for hydraulic bulging and drawing forming of bi-metallic composite pipe. Some researchers employed finite element simulation to perform relevant analysis, good results were obtained [4-6]; In 1976, Krips once studied the process of tube expanding in a sheet for manufacturing heat exchanger [7,8], he proposed an analytical solution for residual contact pressure of such tube expanding in a sheet, his assumptions were an ideal elastic-plastic material model, and the same elastic modulus for both tube and sheet materials. While in 1982, Masashi Takemoto gave the relationship between the expanding pressure $P_{i}$ and the contact between the pressure $P_{c}$ by assuming Tresca yield criterion and ideal elastic-plastic material model [9]. In 1988, Yan proposed an equation of residual contact pressure for heat exchanger without considering strain hardening [10].

In fact, above residual contact pressure formulas are for heat exchanger in expansion process of tube to sheet, which defers from the forming process of bimetallic composite pipe actually. Therefore, the theoretical formulas for tube and sheet expansion for heat exchanger are not connected with the case of plastic forming process of bi-metallic composite pipe integration as a whole. Wang studied the hydraulic expansion process of bi-metallic composite pipe in accordance with the principles of

\footnotetext{
SCHOOL OF CHEMICAL ENGINEERING, NORTHWEST UNIVERSITY, XIÁN, 710069, CHINA

* Corresponding author: mszheng2@yahoo.com
} 
graphic method [11]. But his prediction doesn't agree with his experimental data reasonably.

In the present article, it assumes plane strain assumption and elastic-perfectly plastic material model, so as to analyze the hydro-forming process of bi-metallic composite pipe, and to propose expressions for predicting hydro-forming pressure and residual stress of the forming process in such situation.

\section{Stress analysis in hydro-forming process}

Fig. 1 shows the initial state of the bi-metallic composite pipe system. The inner and outer radii of the base tube are $c$ and $d$, respectively; the inner and outer radii of the liner pipe are $a$ and $b$, respectively, the wall thickness of the liner pipe is $\lambda=b-a$. Before the composite process, $c>b$, i.e., there exists a gap between the liner and outer tubes.

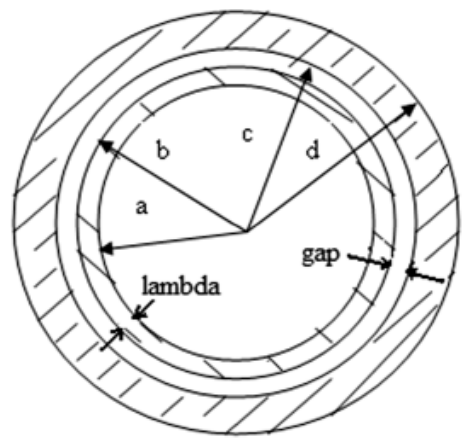

Fig. 1. Schematic diagram of bi-metallic composite pipe

Fig. 2 shows the stress state. According to the elastic-plastic mechanics principle [12], during the bulging forming process, the liner pipe expands gradually due to the action of the inner pressure, and contacts with the outer tube finally.

Assuming elastic-perfectly plastic material model, under conditions of plane strain, the liner pipe deforms plastically and there is no contact pressure until the expanding liner could push the outer base tube to deform. According to Tresca's plastic yielding criterion, the internal pressure inside the liner pipe in such condition is

$$
P_{10}=\frac{2(b-a) \sigma_{s 1}}{b+a}=\frac{2 \lambda \sigma_{s 1}}{b+a}
$$

wherein $\sigma_{s 1}$ is the yield strength of the liner material.

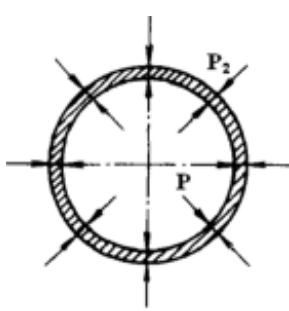

(a)

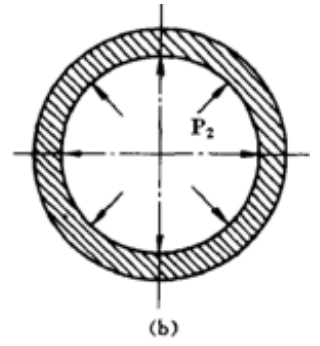

(b)
Fig. 2. Stress state of the bi-metallic composite pipe forming process (a) liner; (b) base tube
There occurs contact pressure between the outer tube and the liner pipe as the inner pipe expands further. If the contact pressure is $P_{2}$, the internal pressure inside the liner pipe is

$$
P=P_{10}+P_{2}
$$

According to elastic-plastic mechanics principle [12], the plastic yielding happens at the inner surface of the base tube as $P_{2}$ reaches to $\frac{\sigma_{s 2}}{2}\left(1-\frac{c^{2}}{d^{2}}\right)$. Therefore, the limit value for $P_{2}$ is

$$
P_{2 c}=\frac{\sigma_{s 2}}{2}\left(1-\frac{c^{2}}{d^{2}}\right)
$$

wherein $\sigma_{s 2}$ is the yield strength of the base tube material.

Simultaneously, the internal pressure inside the liner pipe $P$ (Eq. 2) reaches to its limit $P c$, and describes as:

$$
P_{c}=\frac{2 \lambda \sigma_{s 1}}{b+a}+\frac{\sigma_{s 2}}{2}\left(1-\frac{c^{2}}{d^{2}}\right)
$$

In the bulging process, after the liner pipe contacts with the base pipe and generates contact pressure, the base tube withstands the action of the contact pressure $P 2$. According to elastic-plastic mechanics principle [12], the stresses and the displacement at the position of radius $r$ within the base tube are,

$$
\begin{gathered}
\sigma_{r}=\frac{c^{2} P_{2}}{d^{2}-c^{2}}\left(1-\frac{d^{2}}{r^{2}}\right), \sigma_{\theta}=\frac{c^{2} P_{2}}{d^{2}-c^{2}}\left(1+\frac{d^{2}}{r^{2}}\right), \\
u=\frac{c^{2} P_{2}}{E_{2}\left(d^{2}-c^{2}\right)}\left(\left(1-v_{2}\right) r+\frac{\left(1+v_{2}\right) d^{2}}{r}\right)
\end{gathered}
$$

wherein, $E_{2}$ and $v_{2}$ are elastic modulus and Poisson's ratio of the base pipe material.

According to elastic-plastic mechanics principle [12], the displacement $\kappa$ at the inner wall of the base pipe is

$$
\kappa=u_{c}=\frac{c^{2} P_{2}}{E_{2}\left(d^{2}-c^{2}\right)} \cdot\left[\left(1-v_{2}\right) c+\frac{\left(1+v_{2}\right) d^{2}}{c}\right]
$$

Meanwhile, since the liner pipe expands plastically during expansion, its outer edge reaches to the round of the radius $c+\kappa$, its circumference is $w=2 \pi(c+\kappa)$.

While, in unloading stage, the internal pressure inside the liner pipe disappears, so the liner pipe will shrinks elastically in unloading, the outer edge of the circumference of the liner pipe will shrink to $w^{\prime}=2 \pi(c+\kappa) \times\left(1-\sigma_{s 1} / E_{1}\right)$. Therefore, the outer edge of the circumference of the liner pipe will produce the following radial displacement,

$$
\varsigma=(c+\kappa) \cdot \sigma_{s 1} / E_{1} \approx c \cdot \sigma_{s 1} / E_{1}
$$

wherein, $E_{1}$ is elastic modulus of the lined tube material.

The displacement $\kappa$ at the inner wall of the base pipe in bulging process minus the radial displacement $\zeta$ of liner pipe due to its natural shrinkage during unloading, will gives an overmatching quantity for the liner and outer tubes in the process of unloading procedure. So, according to elastic-plastic mechanics 
principle [12], the residual pressure at the contact surface in unloading status could be written as,

$$
P_{r c}=\frac{\left(\frac{\kappa-\varsigma}{c}\right)}{\left\{\frac{1}{E_{1}} \cdot\left[\frac{c^{2}+\left(c-\lambda^{\prime}\right)^{2}}{c^{2}-\left(c-\lambda^{\prime}\right)^{2}}-v_{1}\right]+\frac{1}{E_{2}} \cdot\left[\frac{d^{2}+c^{2}}{d^{2}-c^{2}}+v_{2}\right]\right\}}
$$

wherein, $\lambda^{\prime}$ is the wall thickness of the liner pipe after expansion, and it meets to $\lambda^{\prime} \cdot\left(c-\lambda^{\prime} / 2\right)=\lambda \cdot(b-\lambda / 2)$ in accordance with volume invariable principle, it yields $\lambda^{\prime} \approx \lambda \cdot b / c$. Then Eq. 8 becomes,

$$
P_{r c}=\frac{\left(\frac{\kappa-\varsigma}{c}\right)}{\left\{\begin{array}{l}
\frac{1}{E_{1}} \cdot\left[\frac{c^{2}+(c-\lambda \cdot b / c)^{2}}{c^{2}-(c-\lambda \cdot b / c)^{2}}-v_{1}\right]+ \\
+\frac{1}{E_{2}} \cdot\left[\frac{d^{2}+c^{2}}{d^{2}-c^{2}}+v_{2}\right]
\end{array}\right\}}
$$

Substituting Eqs. 6 and 7 into 9, it derives,

$$
P_{r c}=\frac{\left\{\begin{array}{l}
\frac{c P_{2}}{E_{2}\left(d^{2}-c^{2}\right)}\left[\left(1-v_{2}\right) c+\frac{\left(1+v_{2}\right) d^{2}}{c}\right] \\
-\sigma_{s 1} / E_{1}
\end{array}\right\}}{\left\{\begin{array}{l}
\frac{1}{E_{1}} \cdot\left[\frac{c^{2}+(c-\lambda \cdot b / c)^{2}}{c^{2}-(c-\lambda \cdot b / c)^{2}}-v_{1}\right]+ \\
+\frac{1}{E_{2}} \cdot\left[\frac{d^{2}+c^{2}}{d^{2}-c^{2}}+v_{2}\right]
\end{array}\right\}}
$$

Thus, the relationship between the residual pressure $P_{r c}$ at the contact surface after unloading and the inner pressure $P$ during loading process could be obtained with the help of Eq. 2,

$$
\begin{aligned}
& P=P_{10}+P_{2}=\frac{2 \lambda \sigma_{s 1}}{b+a}+ \\
& +\left\{\begin{array}{l}
\left.P_{r c} \cdot\left\{\begin{array}{l}
\frac{1}{E_{1}} \cdot\left[\frac{c^{2}+(c-\lambda \cdot b / c)^{2}}{c^{2}-(c-\lambda \cdot b / c)^{2}}-v_{1}\right] \\
+\frac{1}{E_{2}} \cdot\left[\frac{d^{2}+c^{2}}{d^{2}-c^{2}}+v_{2}\right]
\end{array}\right\}+\frac{\sigma_{s 1}}{E_{1}}\right\} \\
\cdot\left\{\frac{E_{2} \cdot\left(1-c^{2} / d^{2}\right)}{\left[\left(1-v_{2}\right) c^{2} / d^{2}+\left(1+v_{2}\right)\right]}\right\}
\end{array}\right.
\end{aligned}
$$

In addition, it can be seen from Eq. 8, that as the composite between the liner pipe and the base pipe is complete, there is residual compressive stress after unloading only if $\kappa \geq \varsigma$. This leads to a following requirement for $P_{2}$ at this time,

$$
P_{2} \geq P_{2 c}^{\prime}=\frac{\sigma_{s 1} \cdot E_{2} \cdot\left(d^{2}-c^{2}\right)}{\left\{E_{1} \cdot\left[\left(1-v_{2}\right) c^{2}+\left(1+v_{2}\right) d^{2}\right]\right\}}
$$

Combing Eq. 3 with Eq. 12 gives following formula,

$$
P_{2 c} \geq P_{2 c} \text { ', i.e., } \frac{\sigma_{s 2}}{\sigma_{s 1}} \geq \frac{2 E_{2}}{E_{1}} /\left[\left(1+v_{2}\right)+\left(1-v_{2}\right) c^{2} / d^{2}\right]
$$

If the elastic modulus and Poisson's ratio of the lined pipe are the same as those of the base tube, Eq. 13 is reduced to,

$$
\sigma_{s 2} \geq \sigma_{s 1} /\left\{1-0.5\left[(1-v)\left(1-\frac{c^{2}}{d^{2}}\right)\right]\right\}
$$

Since $c<d$, Eq. 14 shows that there is a basic conditions $\sigma_{s 2}>\sigma_{s 1}$ for manufacturing bimetallic composite pipe by using bulging method for the lined pipe with the same elastic modulus and Poisson's ratio as those of the base tube material, and the detailed relationship is formula (14).

In addition, the combination of Eq. 1 and Eq. 2 gives

$$
P_{2}=P-P_{10}=P-\frac{2 \lambda \sigma_{s 1}}{b+a}
$$

Substituting Eq. 15 into Eq. 10 gives

$$
P_{r c}=\frac{\left\{\begin{array}{l}
\left(P-\frac{2 \lambda \sigma_{s 1}}{b+a}\right) \\
E_{2}\left(1-c^{2} / d^{2}\right)
\end{array} \cdot\left[\begin{array}{l}
\left(1-v_{2}\right) c^{2} / d^{2}+ \\
+\left(1+v_{2}\right)
\end{array}\right]-\sigma_{s 1} / E_{1}\right\}}{\left\{\begin{array}{l}
\frac{1}{E_{1}} \cdot\left[\frac{c^{2}+(c-\lambda \cdot b / c)^{2}}{c^{2}-(c-\lambda \cdot b / c)^{2}}-v_{1}\right] \\
+\frac{1}{E_{2}} \cdot\left[\frac{d^{2}+c^{2}}{d^{2}-c^{2}}+v_{2}\right]
\end{array}\right\}}
$$

Eq. 16 indicates the relationship between the residual stress and the loaded internal pressure process.

\section{Stress analysis in mechanical drawing process}

As to the manufacture of bi-metallic tube by drawing method, the production process is shown in Fig. 3.

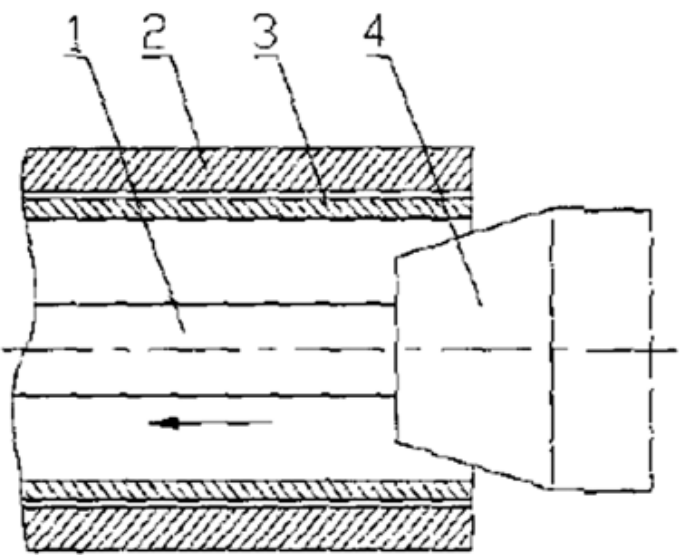

Fig. 3. Manufacture of bi-metallic composite pipe process by drawing method; 1 - drawbar; 2 - base tube; 3 - lined pipe; 4 - extrusion cone

The expressions for inner pressure of bulging the inner pipe, the contact pressure, and the unloading residual pressure $P_{r c}$, Eq. 16 are the same as those in the "hydro-forming process" situation proposed in the previous section. 
According to Coulomb's friction law, there is a sliding friction coefficient $\mu$ of the cone and the lined pipe as the cone moves during the drawing process. The products of $\mu$ and the inner force of the cone acting the inner line pipe gives the friction force, which is equal to the drawing force $F$ for cone to move forward, thus
Wang's experiment shows the test results of residual contact pressure $P_{r c}$ for hydro-forming process under different pressure $P$ conditions [11], which indicated in Fig. 4 by the cross symbol " $\times$ ".

These experimental data could be employed to check the applicable of Eq. (16).

$$
\begin{aligned}
& F=2 \pi c l \mu P=2 \pi c l \mu
\end{aligned}
$$

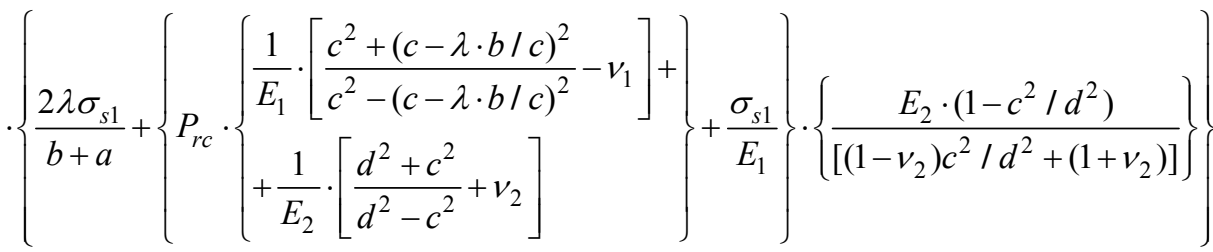

wherein, $l$ is the contact length of the lined pipe and the cone at its maximum cone diameter.

\section{Comparison with experimental data}

In reference [11], Wang et al reported their hydro-forming process in a "self-energizing seal" system, the lined test tube is $144 \times 2 \mathrm{~mm}$ stainless steel tube, the base tube is $159 \times 6 \mathrm{~mm}$ carbon steel pipe, the gap is $\rho=1.5 \mathrm{~mm}$, a length of the pipe is $3000 \mathrm{~mm}$. Table 1 shows the parameters of materials properties.

TABLE 1

Parameters of materials properties

\begin{tabular}{|l|c|c|}
\hline \hline \multicolumn{1}{|c|}{ Name } & Liner pipe & Base pipe \\
\hline Material Grade & 1 Cr18Ni9Ti & $20 \#$ \\
\hline Yield strength $\sigma_{s}(\mathrm{MPa})$ & 213 & 281 \\
\hline Ultimate tensile strength $\sigma_{b}(\mathrm{MPa})$ & 590 & 480 \\
\hline Elastic modulus $E(\mathrm{GPa})$ & 198 & 200 \\
\hline Strain hardening stress $\sigma_{s}{ }^{\prime}(\mathrm{MPa})$ & $246^{\mathrm{a}}$ & \\
\hline Poisson's ratio & 0.3 & 0.3 \\
\hline
\end{tabular}

${ }^{\text {a }}$ The stress in the liner pipe material $\sigma_{s 1}{ }^{\prime}$ at $2.08 \%$ strain is being $246 \mathrm{MPa}$ based on the strain-stress curve of material.
The relevant values of the material parameters shown in Table 1 and yield strength $213 \mathrm{MPa}$ of the liner pipe $1 \mathrm{Cr} 18 \mathrm{Ni} 9 \mathrm{Ti}$ are directly substituting into Eq. 16. It obtains an expression

$$
P_{r c}=0.150 P-2.889, P(\mathrm{MPa})
$$

Fig. 4 also shows the predicted curve of Eq. 18 by the dotted triangle line. As it can be seen from Fig. 4, although the trend of experimental data is consistent with the predicted curve without considering strain hardening, the predicted result of residual stress for the same inner loading pressure is higher than the experimental results.

However, when certain strain hardening for the liner is considered, i.e., taking the strain hardening stress $246 \mathrm{MPa}$ of $1 \mathrm{Cr} 18 \mathrm{Ni}$ Ti as its yield strength and the relevant parameters in Table 1 into Eq. 16, we obtained

$$
P_{r c}=0.150 P-3.336, P(\mathrm{MPa})
$$

Fig. 4 also shows the predicted curve of Eq. 19 by solid line. As can be seen from Fig. 4, the experimental results are agreed with the predicted curve given by Eq. 19 as a certain strain hardening in lined pipe is considered.

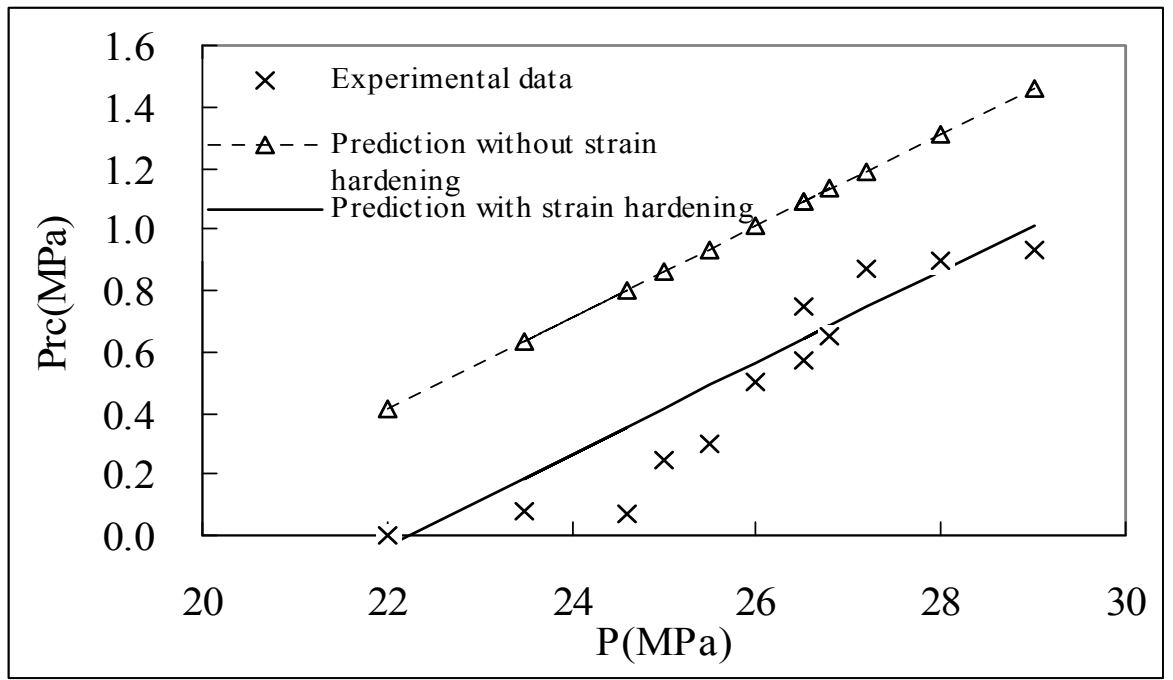

Fig. 4. Comparison of experimental data with predictions 


\section{Conclusion}

In this article, it assumes plane strain conditions and elastic-perfectly plastic material model; the hydraulic bulge forming process of bi-metallic composite pipe is studied, and the relevant formulas are derived; comparing the predictions with experimental results it was shown that the prediction was agreed with the experimental data quite well with taking some strain hardening of liner pipe into consideration.

\section{REFERENCES}

[1] H. Haghighat, G.R. Asgari, A generalized spherical velocity field for bi-metallic tube extrusion through dies of any shape, International Journal of Mechanical Sciences 53, 248-253 (2011).

[2] M. Knezevic, M. Jahedi, Y.P. Korkolis, I.J. Beyerlein, Material-based design of the extrusion of bimetallic tubes, Computational Materials Science 95, 63-73 (2014).

[3] N.R. Chitkara, A. Aleem, Extrusion of axi-symmetric bi-metallic tubes from solid circular billets: application of a generalized upper bound analysis and some experiments, International Journal of Mechanical Sciences 43, 2833-2856 (2001).

[4] R. Lapovok, H.P. Ng, D. Tomus, Y. Estrin, Bimetallic copper aluminium tube by severe plastic deformation, Scripta Materialia 66, 1081-1084 (2012).
[5] X. Yang, F. Sun, Z. Zhang, H. Shen, S. Guo, Optimization of drawing parameters for copper tubes with hollow sinking based on FEM simulation, The Chinese Journal of Nonferrous Metals 18, 2245-2252 (2008).

[6] L. Xue, Y. He, R. Liu, C. Dai, J. Chen, FEA on empty-sunken steel tube based on ANSYS/LSDYNA, Journal of Plasticity Engineering 12 (5), 74-77 (2005).

[7] K. Vedeld, H. Osnes, O. Fyrileiv, Analytical expressions for stress distributions in lined pipes: Axial stress and contact pressure interaction, Marine Structures 26, 1-26 (2012).

[8] H. Krips, M. Podhorsky, Hyraolic Expansion a New Method for Anchoring of Tubes, VGB KRAFWERKSTECHINK 56 (7), 144-153 (1976)

[9] M. Takemoto, Tubular Heat Exchanger strength - hydraulic expanding fitting pull-off force detained, Pressure Vessel 2, 68-75 (1984).

[10] H. Yan, C. Yu, Drawing algorithm for residual contact pressure in heat exchangers during hydraulic expanding, Chemical Machinery 28, 211-214 (2001).

[11] X. Wang, P. Li, R. Wang, Study on hydro-forming technology of manufacturing bimetallic CRA-lined pipe, Int. J. of Machine Tools \& Manuf. 45, 373-378 (2005).

[12] B. Xu, X. Liu, Applied elastic-plastic mechanics, Tsinghua University Press, 128-233 (1995). 